\title{
Sliding Mode Observers Based-Simultaneous Actuator and Sensor Faults Estimation for Linear Parameter Varying Systems
}

\author{
Ali Ben Brahim, Slim Dhahri, Fayçal Ben Hmida, and Anis Sallami \\ Engineering Laboratory of Industrial Systems and Renewable Energies, National Higher School of Engineers of Tunis, \\ University of Tunis, Tunis, Tunisia \\ Correspondence should be addressed to Ali Ben Brahim; alibenibrahimm@yahoo.com
}

Received 3 June 2017; Revised 28 September 2017; Accepted 29 October 2017; Published 4 December 2017

Academic Editor: Sebastian Heidenreich

Copyright (C) 2017 Ali Ben Brahim et al. This is an open access article distributed under the Creative Commons Attribution License, which permits unrestricted use, distribution, and reproduction in any medium, provided the original work is properly cited.

\begin{abstract}
This paper proposes a scheme to estimate actuator and sensor faults simultaneously for a class of linear parameter varying system expressed in polytopic structure where its parameters evolve in the hypercube domain. Transformed coordinate system design is adopted to decouple faults in actuators and sensors during the course of the system's operation coincidentally, and then two polytopic subsystems are constructed. The first subsystem includes the effect of actuator faults but is free from sensor faults and the second one is affected only by sensor faults. The main contribution is to conceive two polytopic sliding mode observers in order to estimate the system states and actuator and sensor faults at the same time. Meanwhile, in linear matrix inequality optimization formalism, sufficient conditions are derived with $H_{\infty}$ performances to guarantee the stability of estimation error and to minimize the effect of disturbances. Therefore, all parameters of observers can be designed by solving these conditions. Finally, simulation results are given to illustrate the effectiveness of the proposed simultaneous actuator and sensor faults estimation.
\end{abstract}

\section{Introduction}

Fault disturbs the normal system operations, thus causing an unacceptable deterioration of these performances or leading to wrong dangerous situations. Fault can occur in any part of the system such as actuators and sensors. Sensor fault degrades the feedback system performances and so touches the control stability. Similarly, actuator fault may lead to minimizing the performances of design controllers or even the overall system execution. To this end, determining the size, location, and dynamic behavior of the fault along a system trajectories becomes a powerful alternative active research. Indeed, fault estimation is considered a major problem in the modern control theory that received a considerable amount of attention during the past few years. In the context of actuator faults estimation, constructing diagnosis model in order to estimate faults is not possible if sensor faults occur simultaneously. The same difficulty is present when trying to estimate sensor faults. Several design methods have been developed in a precise and effective way when actuator and sensor faults estimation is divided into two steps.
Step 1. Actuator faults estimation is proven without considering sensor faults [1-11].

Step 2. Sensor faults estimation is solved without considering actuator faults [12-19].

Nevertheless, in practical systems, it is often the case when actuator and sensor faults occur simultaneously. In this framework, simultaneous faults estimation is highly important. So far, [20] considers the problem of simultaneous actuator and sensor fault estimation for Lipschitz nonlinear systems. The idea of this work based on the transformation of sensor fault obtained an augmented actuator fault vector. In [21], the authors propose simultaneously estimating actuator and sensor faults study. Two subsystems are constructed where each one contains a particular class of faults (actuator or sensor). More recently, $[22,23]$ consider the problem of robust simultaneous actuator and sensor faults estimation study for a class of uncertain Takagi-Sugeno nonlinear system with unmeasurable premise variables. The main contribution is to develop a sliding mode observer (SMO) with two 
discontinuous terms to solve the problem of simultaneous faults.

On the other hand, very interesting approaches have represented actual physical systems under linear parameter varying (LPV) representation expressed in polytopic structure where its parameters evolve in the hypercube domain. Roughly speaking, the feature is to understand the overall system behavior by a set of local linear models scheduled by convex weighting functions. Taking the polytopic LPV representation, several attempts have been oriented to the diagnosis of nonlinear systems; see, for instance, [24-28]. More recently, [29] addresses the problem of simultaneous actuator and sensor faults estimation for the polytopic LPV systems. The idea is based on the descriptor approach by extending the sensor faults as an auxiliary states vector. This approach consists in transforming a standard LPV system into a descriptor form to estimate sensor faults. Furthermore, this approach is not applicable directly to real descriptor systems; the fact is that these systems have more complicated structure and usually have three types of modes: dynamic, impulse, and static, which are not considered in this approach. Reference [30] investigates simultaneous actuator and sensor faults estimation for polytopic LPV systems by introducing a filter in order to transform sensor faults as a "pseudoactuator" faults. Appropriate filtering of the original system output yields "augmented fictitious systems" with augmented actuator faults comprising actuator and sensor faults. Nevertheless, this leads to some conservatism. This approach is based on some rank restrictive assumptions, which must be verified for both original and augmented systems to ensure the observer-based fault estimation.

Regarding the fact that simultaneous actuator and sensor faults scenario has not yet been fully tackled, we will develop robust fault estimation scheme for LPV system subject to both faults affecting actuators and sensor faults and disturbances in the present study. It should be noticed that the LPV system is expressed in polytopic structure where its parameters evolve in the hypercube domain. Motivated by the success of SMO based methods of fault estimation, the present study focuses on developing effective and robust simultaneous actuator and sensor faults estimation for a class of LPV systems. The main contributions are as follows:

(i) Designing state and output coordinate transformation such that actuator and sensor faults are completely separated, hence two polytopic LPV subsystems being introduced where each one contains only a particular class of faults (actuator or sensor).

(ii) Constructing of two sliding mode observers, thanks to their robustness against disturbances, which are designed to exactly simultaneous polytopic LPV system states, faults affecting actuators, and sensor faults estimation.

(iii) Developing convex Linear Matrix Inequalities (LMIs) optimization approach in which the disturbance attenuation level is minimized

The outline of this paper is organized as follows: in Section 2, we describe the uncertain LPV system expressed by the polytopic representation. In Section 3, we propose the $H_{\infty}$ faults estimation scheme using two sliding mode observers. Section 4 is devoted to simultaneous actuator and sensor faults estimation. Simulation examples are described in Section 5, illustrating the effectiveness of the proposed method. Finally, Section 6 presents some concluding remarks.

\section{Problem Formulation}

Consider the LPV system governed by

$$
\begin{aligned}
\dot{x}(t)= & A(\theta(t)) x(t)+B(\theta(t)) u(t)+D(\theta(t)) \xi(t) \\
& +M(\theta(t)) f_{a}(t), \\
y(t)= & C(\theta(t)) x(t)+N(\theta(t)) f_{s}(t),
\end{aligned}
$$

where $x(t) \in \mathrm{R}^{n}$ is the state vector, $u(t) \in \mathrm{R}^{m}$ is the control input, and $y(t) \in \mathrm{R}^{p}$ denotes the output vector. $f_{a}(t) \epsilon$ $\mathrm{R}^{q}$ and $f_{s}(t) \in \mathrm{R}^{h}$ represent actuator and sensor faults, respectively, which are assumed unknown but bounded by known constants such as $\left\|f_{a}(t)\right\| \leq \rho_{1}$ and $\left\|f_{s}(t)\right\| \leq \rho_{2}$. $\xi(t) \in \mathrm{R}^{l}$ stands for the unknown disturbances such that $\|\xi(t)\| \leq \xi_{0}$.

$A(\theta(t)) \in \mathrm{R}^{n \times n}, B(\theta(t)) \in \mathrm{R}^{n \times m}, M(\theta(t)) \in \mathrm{R}^{n \times q}$, $D(\theta(t)) \in \mathrm{R}^{n \times l}, C(\theta(t)) \in \mathrm{R}^{p \times n}$, and $N(\theta(t)) \in \mathrm{R}^{p \times h}$ are continuous functions that depend affinely on the timevarying parameter vector $\theta(t) \in \mathrm{R}^{v}$ where $n>p \geq q$.

In what follows, the parameter vector $\theta(t)$ is considered bounded and lies into a hypercube $\Theta$ as

$$
\theta(t) \in \Theta=\{\theta \mid \underline{\theta}(t) \leq \theta(t) \leq \bar{\theta}(t)\}, \quad \forall t \geq 0 .
$$

Based on parameter affine dependence [25, 28], the elements of the representation (1) can be written as

$$
\Pi(\theta(t))=\Pi_{0}+\sum_{i=1}^{k} \theta_{i}(t) \Pi_{i}, \quad \forall \theta(t) \in \Theta,
$$

where

$$
\Pi(\theta(t))=\left[\begin{array}{cccc}
A(\theta(t)) & B(\theta(t)) & D(\theta(t)) & M(\theta(t)) \\
C(\theta(t)) & 0 & 0 & N(\theta(t))
\end{array}\right] .
$$

Consequently, the LPV system (1) can be transformed into a convex interpolation of the vertices of $\Theta$ such as

$$
\begin{aligned}
& \dot{x}(t) \\
& \quad=\sum_{i=1}^{k} \mu_{i}(\theta(t))\left\{A_{i} x(t)+B_{i} u(t)+D_{i} \xi(t)+M_{i} f_{a}(t)\right\} \\
& y(t)=\sum_{i=1}^{k} \mu_{i}(\theta(t))\left\{C_{i} x(t)+N_{i} f_{s}(t)\right\}
\end{aligned}
$$

where $A_{i}, B_{i}, D_{i}, M_{i}, C_{i}$, and $N_{i}$ are time invariant known matrices with appropriate dimensions defined for the $i$ th vertex of $\Theta$. 
$\mu_{i}(\theta(t))$ denotes the weighting functions which vary into the convex set $\Omega$ :

$$
\begin{aligned}
\Omega & =\left\{\mu(\theta(t))=\left[\begin{array}{lll}
\mu_{1}(\theta(t)) & \cdots & \mu_{k}(\theta(t))
\end{array}\right]^{T}, \mu(\theta(t))\right. \\
& \left.\geq 0, \sum_{i=1}^{k} \mu_{i}(\theta(t))=1\right\} .
\end{aligned}
$$

Herein, $k=2^{v}$ is the total number of subsystems. It is noted that system (5) refers to a polytopic LPV system where its parameters evolve in the hypercube $\Theta$. Each local model must verify the following assumptions.

Assumption A1. The actuator fault distribution matrix $M_{i}$ is of full-column rank, $\forall i \in[1, \ldots, k]$, that is,

$$
\operatorname{rank}\left(C_{i} M_{i}\right)=\operatorname{rank}\left(M_{i}\right)=q .
$$

Assumption A2. System (5) is with minimum phase; that is, the invariant zeros of the triplet $\left(A_{i}, C_{i}, M_{i}\right), \forall i \in[1, \ldots, k]$, are all in the open left-hand complex plant, or

$$
\operatorname{rank}\left[\begin{array}{cc}
s I_{n}-A_{i} & M_{i} \\
C_{i} & 0
\end{array}\right]=n+q
$$

holds for all complex number $s$ with $\operatorname{Re}(s) \geq 0$.

Assumptions A1 and A2 imply that the system is of relative degree one and minimum phase. These conditions are necessary and sufficient for the sliding mode observer design based simultaneous fault estimation.

Under Assumption A1, there exists linear transformation [11] such that the polytopic LPV system matrices from (5) yield, $\forall i \in[1, \ldots, k]$,

$$
\begin{aligned}
& T_{i} A_{i} T_{i}^{-1}=\left[\begin{array}{ll}
A_{1, i} & A_{2, i} \\
A_{3, i} & A_{4, i}
\end{array}\right], \\
& T_{i} B_{i}=\left[\begin{array}{c}
B_{1, i} \\
B_{2, i}
\end{array}\right] \text {, } \\
& T_{i} D_{i}=\left[\begin{array}{l}
D_{1, i} \\
D_{2, i}
\end{array}\right] \text {, } \\
& T_{i} M_{i}=\left[\begin{array}{c}
M_{1, i} \\
0
\end{array}\right] \text {, } \\
& S_{i} C_{i} T_{i}^{-1}=\left[\begin{array}{cc}
C_{1, i} & 0 \\
0 & C_{4, i}
\end{array}\right] \text {, } \\
& S_{i} N_{i}=\left[\begin{array}{c}
0 \\
N_{2, i}
\end{array}\right] \text {, }
\end{aligned}
$$

where

$$
\begin{aligned}
T_{i} & =\left[\begin{array}{ll}
T_{1, i}^{T} & T_{2, i}^{T}
\end{array}\right]^{T} \in \mathrm{R}^{n \times n}, \\
S_{i} & =\left[\begin{array}{ll}
S_{1, i}^{T} & S_{2, i}^{T}
\end{array}\right]^{T} \in \mathrm{R}^{p \times p}, \\
A_{1, i} & \in \mathrm{R}^{q \times q}, \\
B_{1, i} & \in \mathrm{R}^{q \times m},
\end{aligned}
$$

$$
\begin{aligned}
& M_{1, i} \in \mathrm{R}^{q \times q}, \\
& D_{1, i} \in \mathrm{R}^{q \times l}, \\
& C_{1, i} \in \mathrm{R}^{q \times q}, \\
& C_{4, i} \in \mathrm{R}^{(p-q) \times(n-q)}, \\
& N_{2, i} \in \mathrm{R}^{(p-q) \times h},
\end{aligned}
$$

$\operatorname{rank}\left(M_{1, i}\right)=q$ and $C_{1, i}$ is invertible. The matrices $N_{2, i}$ have a structure $N_{2, i}=\left[\begin{array}{c}0 \\ N_{22, i}\end{array}\right]$ with $N_{22, i} \in \mathrm{R}^{h \times h}$.

Lemma 1. The pair matrix $\left(A_{4, i}, C_{4, i}\right)$ is detectable, $\forall i \in$ $[1, \ldots, k]$, if and only if each local model for the polytopic $L P V$ system (5) is minimum phase; that is, Assumption A2 holds.

Proof (see [21]). Based on the preceding state and output transformations, $\left[\begin{array}{lll}x_{1}^{T}(t) & x_{2}^{T}(t)\end{array}\right]^{T}=T_{i} x(t)$ and $\left[\begin{array}{ll}y_{1}^{T}(t) & y_{2}^{T}(t)\end{array}\right]^{T}=S_{i} y(t)$, the polytopic LPV system (5) is converted into two submodels:

$$
\begin{aligned}
& \dot{x}_{1}(t)=\sum_{i=1}^{k} \mu_{i}(\theta(t))\left\{A_{1, i} x_{1}(t)+A_{2, i} x_{2}(t)+B_{1, i} u(t)\right. \\
& \left.+D_{1, i} \xi(t)+M_{1, i} f_{a}(t)\right\}, \\
& y_{1}(t)=\sum_{i=1}^{k} \mu_{i}(\theta(t))\left\{C_{1, i} x_{1}(t)\right\}, \\
& \dot{x}_{2}(t)=\sum_{i=1}^{k} \mu_{i}(\theta(t))\left\{A_{3, i} x_{1}(t)+A_{4, i} x_{2}(t)+B_{2, i} u(t)\right. \\
& \left.D_{2, i} \xi(t)\right\}, \\
& y_{2}(t)=\sum_{i=1}^{k} \mu_{i}(\theta(t))\left\{C_{4, i} x_{2}(t)+N_{2, i} f_{s}(t)\right\},
\end{aligned}
$$

where (11) is referred to as the first subsystem which contains only actuator faults $f_{a}(t)$ and (12) is referred to as the second subsystem which results in only sensor faults $f_{s}(t)$.

We define a new system state vector $x_{3}(t)=\int_{0}^{t} y_{2}(\tau) d \tau$ where $\dot{x}_{3}(t)=\sum_{i=1}^{k} \mu_{i}(\theta(t))\left\{C_{4, i} x_{2}(t)+N_{2, i} f_{s}(t)\right\}$. Thus, an augmented polytopic LPV submodel of order $(n+p-2 q)$ can be expressed as

$$
\begin{aligned}
\dot{x}_{0}(t) & =\sum_{i=1}^{k} \mu_{i}(\theta(t))\left\{A_{0, i} x_{0}(t)+A_{01, i} x_{1}(t)+B_{0, i} u(t)\right. \\
+ & \left.D_{0, i} \xi(t)+M_{0, i} f_{s}(t)\right\}, \\
y_{0}(t) & =\sum_{i=1}^{k} \mu_{i}(\theta(t))\left\{C_{0, i} x_{0}(t)\right\},
\end{aligned}
$$

such that

$$
\begin{aligned}
& x_{0}(t)=\left[\begin{array}{l}
x_{2}(t) \\
x_{3}(t)
\end{array}\right] \in \mathrm{R}^{n+p-2 q}, \\
& y_{0}(t) \in \mathrm{R}^{p-q}
\end{aligned}
$$




$$
\begin{aligned}
A_{0, i} & =\left[\begin{array}{ll}
A_{4, i} & 0 \\
C_{4, i} & 0
\end{array}\right] \in \mathrm{R}^{(n+p-2 q) \times(n+p-2 q)}, \\
A_{01, i} & =\left[\begin{array}{c}
A_{3, i} \\
0
\end{array}\right] \in \mathrm{R}^{(n+p-2 q) \times q}, \\
B_{0, i} & =\left[\begin{array}{c}
B_{2, i} \\
0
\end{array}\right] \in \mathrm{R}^{(n+p-2 q) \times m}, \\
D_{0, i} & =\left[\begin{array}{c}
D_{2, i} \\
0
\end{array}\right] \in \mathrm{R}^{(n+p-2 q) \times l}, \\
M_{0, i} & =\left[\begin{array}{c}
0 \\
N_{2, i}
\end{array}\right] \in \mathrm{R}^{(n+p-2 q) \times h}, \\
C_{0, i} & =\left[\begin{array}{ll}
0 & I_{p-q}
\end{array}\right] \in \mathrm{R}^{(p-q) \times(n+p-2 q)} .
\end{aligned}
$$

The following lemma express the observability properties of the polytopic LPV subsystem (13).

Lemma 2. The pair $\left(A_{0, i}, C_{0, i}\right)$ is observable, $\forall i \in[1, \ldots, k]$, if the pair $\left(A_{4, i}, C_{4, i}\right)$ is detectable, $\forall i \in[1, \ldots, k]$. Then, there exists matrix $L$, having the special structure $L=\left[\begin{array}{ll}L_{1} & 0\end{array}\right]$, such that $A_{4, i}+L C_{4, i}$ is stable $\forall i \in[1, \ldots, k]$.

Proof (see [21]). Consider system (13). Introduce a coordinate transformation $z(t)=T_{L} x_{0}(t)$ where

$$
T_{L}:=\left[\begin{array}{cc}
I_{n-q} & L \\
0 & I_{p-q}
\end{array}\right] .
$$

It follows that, in the new coordinate system $z(t)$, system (13) becomes

$$
\begin{aligned}
& \dot{z}(t)=\sum_{i=1}^{k} \mu_{i}(\theta(t))\left\{A_{z, i} z(t)+T_{L} A_{01, i} x_{1}(t)+B_{z, i} u(t)\right. \\
& \left.+D_{z, i} \xi(t)+M_{z, i} f_{s}(t)\right\}, \\
& y_{0}(t)=\sum_{i=1}^{k} \mu_{i}(\theta(t))\left\{C_{z, i} z(t)\right\},
\end{aligned}
$$

where

$$
\begin{aligned}
A_{z, i} & =\left[\begin{array}{cc}
A_{4, i}+L C_{4, i} & -\left(A_{4, i}+L C_{4, i}\right) L \\
C_{4, i} & -C_{4, i} L
\end{array}\right], \\
B_{z, i} & =\left[\begin{array}{c}
B_{2, i} \\
0
\end{array}\right], \\
D_{z, i} & =\left[\begin{array}{c}
D_{2, i} \\
0
\end{array}\right], \\
M_{z, i} & =\left[\begin{array}{c}
0 \\
N_{2, i}
\end{array}\right], \\
C_{z, i} & =\left[\begin{array}{ll}
0 & I_{p-q}
\end{array}\right] .
\end{aligned}
$$

The following section is dedicated to the design of the main results for this paper.

\section{Sliding Mode Observers Design}

We propose to conceive two sliding mode observers for the polytopic LPV system (5) expressed in two subsystems (11) and (16) based simultaneous actuator and sensor faults estimation design with minimizing the effect of disturbances using $H_{\infty}$ performances.

As a result, in the new coordinate systems $z(t),(11)$ and (16) can be rewritten, respectively, as

$$
\begin{aligned}
& \dot{x}_{1}(t)=\sum_{i=1}^{k} \mu_{i}(\theta(t))\left\{A_{1, i} x_{1}(t)+A_{2, i} z_{1}(t)+B_{1, i} u(t)\right. \\
& \left.-A_{2, i} L y_{0}(t)+D_{1, i} \xi(t)+M_{1, i} f_{a}(t)\right\}, \\
& y_{1}(t)=\sum_{i=1}^{k} \mu_{i}(\theta(t))\left\{C_{1, i} x_{1}(t)\right\}, \\
& \dot{z}_{1}(t)=\sum_{i=1}^{k} \mu_{i}(\theta(t))\left\{\left(A_{4, i}+L C_{4, i}\right) z_{1}(t)\right. \\
& \quad\left(A_{4, i}+L C_{4, i}\right) L z_{2}(t)+A_{3, i} x_{1}(t)+B_{2, i} u(t) \\
& \left.+D_{2, i} \xi(t)\right\}, \\
& \dot{z}_{2}(t)=\sum_{i=1}^{k} \mu_{i}(\theta(t))\left\{C_{4, i} z_{1}(t)-C_{4, i} L z_{2}(t)\right. \\
& \left.+N_{2, i} f_{s}(t)\right\}, \\
& y_{0}(t)=z_{2}(t) .
\end{aligned}
$$

For subsystems (18) and (19), we construct the following two sliding mode observers, respectively,

$$
\begin{aligned}
& \dot{\hat{x}}_{1}(t)=\sum_{i=1}^{k} \mu_{i}(\theta(t))\left\{A_{1, i} \widehat{x}_{1}(t)+A_{2, i} \widehat{z}_{1}(t)\right. \\
& \quad-A_{2, i} L y_{0}(t)+B_{1, i} u(t)+M_{1, i} v_{1, i}(t) \\
& \left.+\left(A_{1, i}-A_{1, i}^{s}\right) C_{1, i}^{-1}\left(y_{1}(t)-\widehat{y}_{1}(t)\right)\right\} \\
& \widehat{y}_{1}(t)=\sum_{i=1}^{k} \mu_{i}(\theta(t))\left\{C_{1, i} \widehat{x}_{1}(t)\right\} \\
& \dot{\vec{z}}_{1}(t)=\sum_{i=1}^{k} \mu_{i}(\theta(t))\left\{\left(A_{4, i}+L C_{4, i}\right) \widehat{z}_{1}(t)\right. \\
& \quad-\left(A_{4, i}+L C_{4, i}\right) L y_{0}(t)+B_{2, i} u(t) \\
& \left.+A_{3, i} C_{1, i}^{-1} y_{1}(t)\right\} \\
& \dot{\widehat{z}}_{2}(t)=\sum_{i=1}^{k} \mu_{i}(\theta(t))\left\{C_{4, i} \widehat{z}_{1}(t)-C_{4, i} L \widehat{z}_{2}(t)\right. \\
& \left.\quad-\left(C_{4, i} L+K_{i}\right)\left(y_{0}(t)-\widehat{y}_{0}(t)\right)+N_{2, i} v_{2, i}(t)\right\} \\
& \widehat{y}_{0}(t)=\widehat{z}_{2}(t) \\
& A_{1, i}^{s} \in \mathrm{R}^{q \times q} \text { and } K_{i} \in \mathrm{R}^{(p-q) \times(p-q)} \text { are the observer gains. }
\end{aligned}
$$


The discontinuous output error injection signals $v_{1, i}(t)$ and $v_{2, i}(t)$ are expressed as, respectively,

$$
\begin{aligned}
& v_{1, i}(t)= \begin{cases}\left(\rho_{1}+\eta_{1, i}\right) \frac{M_{1, i}^{T} P_{1}\left(C_{1, i}^{-1} S_{1, i} y(t)-\widehat{x}_{1}(t)\right)}{\left\|M_{1, i}^{T} P_{1}\left(C_{1, i}^{-1} S_{1, i} y(t)-\widehat{x}_{1}(t)\right)\right\|} & \text { if }\left(C_{1, i}^{-1} S_{1, i} y(t)-\widehat{x}_{1}(t)\right) \neq 0 \\
0 & \text { otherwise, }\end{cases} \\
& v_{2, i}(t)= \begin{cases}\left(\rho_{2}+\eta_{2, i}\right) \frac{N_{2, i}^{T} \bar{P}_{03}\left(y_{0}(t)-\widehat{y}_{0}(t)\right)}{\left\|N_{2, i}^{T} \bar{P}_{03}\left(y_{0}(t)-\widehat{y}_{0}(t)\right)\right\|} & \text { if }\left(y_{0}(t)-\widehat{y}_{0}(t)\right) \neq 0 \\
0 & \text { otherwise, }\end{cases}
\end{aligned}
$$

where $P_{1} \in \mathrm{R}^{q \times q}$ and $\bar{P}_{03} \in \mathrm{R}^{(p-q) \times(p-q)}$ are symmetric definite Lyapunov matrices. $\eta_{1, i}$ and $\eta_{2, i}$ are two positive scalars to be determined.

Let $e_{1}(t)=x_{1}(t)-\widehat{x}_{1}(t), e_{2}(t)=z_{1}(t)-\widehat{z}_{1}(t)$, and $e_{3}(t)=z_{2}(t)-\widehat{z}_{2}(t)$. Then, from (18)-(19) and (20)-(21), the state estimation errors dynamics are described by

$$
\begin{aligned}
\dot{e}_{1}(t) & =\sum_{i=1}^{k} \mu_{i}(\theta(t))\left\{A_{1, i}^{s} e_{1}(t)+A_{2, i} e_{2}(t)+D_{1, i} \xi(t)\right. \\
+ & \left.M_{1, i} f_{a}(t)-M_{1, i} v_{1, i}(t)\right\}, \\
\dot{e}_{2}(t) & =\sum_{i=1}^{k} \mu_{i}(\theta(t))\left\{\left(A_{4, i}+L C_{4, i}\right) e_{2}(t)+D_{2, i} \xi(t)\right\}, \\
\dot{e}_{3}(t) & =\sum_{i=1}^{k} \mu_{i}(\theta(t))\left\{C_{4, i} e_{2}(t)+K_{i} e_{3}(t)+N_{2, i} f_{s}(t)\right. \\
- & \left.N_{2, i} v_{2, i}(t)\right\} .
\end{aligned}
$$

The objective now is to present the sufficient conditions for the stability with $H_{\infty}$ performances of the observer errors (24)-(26) by using Lyapunov stability and LMIs technique.

\subsection{Sliding Motion Stability. Suppose that}

$$
g(t)=H e(t)=H\left[\begin{array}{l}
e_{1}(t) \\
e_{2}(t) \\
e_{3}(t)
\end{array}\right]
$$

is a linear error variables rearrangement. $H$ is a prespecified weight matrix and assumed to have full rank:

$$
H:=\left[\begin{array}{ccc}
H_{1} & 0 & 0 \\
0 & H_{2} & 0 \\
0 & 0 & H_{3}
\end{array}\right] .
$$

The following theorem provides sufficient conditions to ensure the properties of sliding motion stability with specified $H_{\infty}$ performances to be defined as

$$
\|H\|_{\infty}=\sup _{\|\xi(t)\|_{L 2} \neq 0} \frac{\|g(t)\|_{L 2}^{2}}{\|\xi(t)\|_{L 2}^{2}} \leq \gamma,
$$

where $\gamma$ is a small positive constant. So that, small $H_{\infty}$ gain of the transfer function from the disturbances $\xi(t)$ to the estimation errors combination $g(t)$ means a small influence of $\xi(t)$ on $e(t)$.

Theorem 3. Consider the polytopic LPV systems (18)-(19) and the sliding mode observers (20)-(21). The observer estimation errors (24)-(26) are stable with attenuation level $\gamma>0$ subject to $\|g(t)\|_{L 2}<\sqrt{\gamma}\|\xi(t)\|_{L 2}$, if there exist the matrices $P_{1}>0$, $P_{01} \in \mathrm{R}^{(n-q) \times(n-q)}>0, \bar{P}_{03}>0, X_{1, i}<0$ such that the following optimization problem holds, $\forall i \in[1, \ldots, k]$,

$$
\begin{array}{clcc}
\operatorname{minimize} & \gamma \\
\text { s.t. } & {\left[\begin{array}{cccc}
X_{1, i}+X_{1, i}^{T}+H_{1}^{T} H_{1} & P_{1} A_{2, i} & 0 & P_{1} D_{1, i} \\
A_{2, i}^{T} P_{1} & X_{2, i}+Y C_{4, i}+C_{4, i}^{T} Y^{T}+H_{2}^{T} H_{2} & C_{4, i}^{T} \bar{P}_{03} & P_{01} D_{2, i} \\
0 & \bar{P}_{03} C_{4, i} & X_{3, i}+X_{3, i}^{T}+H_{3}^{T} H_{3} & 0 \\
D_{1, i}^{T} P_{1} & D_{2, i}^{T} P_{01} & 0 & -\sqrt{\gamma} I_{l}
\end{array}\right]<0,}
\end{array}
$$

where $X_{1, i}=P_{1} A_{1, i}^{s}, X_{2, i}=A_{4, i}^{T} P_{01}+P_{01} A_{4, i}$. 
Once the LMI problem is solved, it can provide design freedom sliding mode observers (20)-(21), such that

$$
\begin{gathered}
L=P_{01}^{-1} Y, \\
A_{1, i}^{s}=P_{1}^{-1} X_{1, i}, \\
K_{i}=\bar{P}_{03}^{-1} X_{3, i} .
\end{gathered}
$$

Proof. The proof of this theorem is performed by using the following Lyapunov function:

$$
V(t)=V_{1}(t)+V_{2}(t)+V_{3}(t)
$$

where $V_{1}(t)=e_{1}^{T}(t) P_{1} e_{1}(t), V_{2}(t)=e_{2}^{T}(t) P_{01} e_{2}(t), V_{3}(t)=$ $e_{3}^{T}(t) \bar{P}_{03} e_{3}(t)$.

The time derivation of $V_{1}(t)$ along a system trajectories is expressed as

$$
\begin{aligned}
& \dot{V}_{1}(t)=\sum_{i=1}^{k} \mu_{i}(\theta(t)) \\
& \cdot\left\{e_{1}^{T}(t)\left(\left(A_{1, i}^{s}\right)^{T} P_{1}+P_{1} A_{1, i}^{s}\right) e_{1}(t)\right. \\
& +2 e_{1}^{T}(t) P_{1} A_{2, i} e_{2}(t)+2 e_{1}^{T}(t) P_{1} D_{1, i} \xi(t) \\
& \left.+2 e_{1}^{T}(t) P_{1} M_{1, i}\left(f_{a}(t)-v_{1, i}(t)\right)\right\} .
\end{aligned}
$$

From the definition of $v_{1, i}(t)$ in (22) and using the bound of $f_{a}(t)$, it follows that

$$
\begin{aligned}
e_{1}^{T}(t) & P_{1} M_{1, i}\left(f_{a}(t)-v_{1, i}(t)\right) \\
= & e_{1}^{T}(t) P_{1} M_{1, i} f_{a}(t) \\
& -\left(\rho_{1}+\eta_{1, i}\right) e_{1}^{T}(t) P_{1} M_{1, i} \frac{M_{1, i}^{T} P_{1} e_{1}(t)}{\left\|M_{1, i}^{T} P_{1} e_{1}(t)\right\|} \\
\leq & -\eta_{1, i}\left\|M_{1, i}^{T} P_{1} e_{1}(t)\right\|<0 .
\end{aligned}
$$

Thus,

$$
\begin{aligned}
& \dot{V}_{1}(t) \leq \sum_{i=1}^{k} \mu_{i}(\theta(t)) \\
& \cdot\left\{e_{1}^{T}(t)\left(\left(A_{1, i}^{s}\right)^{T} P_{1}+P_{1} A_{1, i}^{s}\right) e_{1}(t)\right. \\
& \left.+2 e_{1}^{T}(t) P_{1} A_{2, i} e_{2}(t)+2 e_{1}^{T}(t) P_{1} D_{1, i} \xi(t)\right\} .
\end{aligned}
$$

Likewise, the time derivations of $V_{2}(t)$ and $V_{3}(t)$ along a system trajectories take the following equalities:

$$
\begin{aligned}
& \dot{V}_{2}(t)=\sum_{i=1}^{k} \mu_{i}(\theta(t))\left\{e_{2}^{T}(t)\right. \\
& \cdot\left(\left(A_{4, i}+L C_{4, i}\right)^{T} P_{01}+P_{01}\left(A_{4, i}+L C_{4, i}\right)\right) e_{2}(t) \\
& \left.+2 e_{2}^{T}(t) P_{01} D_{2, i} \xi(t)\right\}, \\
& \dot{V}_{3}(t)=\sum_{i=1}^{k} \mu_{i}(\theta(t))\left\{e_{3}^{T}(t)\left(\bar{P}_{03} K_{i}+K_{i}^{T} \bar{P}_{03}\right) e_{3}(t)\right. \\
& +2 e_{3}^{T}(t) \bar{P}_{03} C_{4, i} e_{2}(t)+2 e_{3}^{T}(t) \bar{P}_{03} N_{2, i} f_{s}(t) \\
& \left.-2 e_{3}^{T}(t) \bar{P}_{03} N_{2, i} v_{2, i}(t)\right\} .
\end{aligned}
$$

Manipulating the definition of $v_{2, i}(t)$ in (23) and using the bound of $f_{s}(t)$, it turns out that

$$
\begin{aligned}
& e_{3}^{T}(t) \bar{P}_{03} N_{2, i}\left(f_{s}(t)-v_{2, i}(t)\right) \\
& \quad \leq-\eta_{2, i}\left\|N_{2, i}^{T} \bar{P}_{03}\left(y_{0}(t)-\widehat{y}_{0}(t)\right)\right\|<0 .
\end{aligned}
$$

Therefore, the time derivation of $V(t)$ is handled as

$$
\begin{aligned}
\dot{V}( & t)=\dot{e}_{1}^{T}(t) P_{1} e_{1}(t)+e_{1}^{T}(t) P_{1} \dot{e}_{1}(t)+\dot{e}_{2}^{T}(t) P_{01} e_{2}(t) \\
+ & e_{2}^{T}(t) P_{01} \dot{e}_{2}(t)+\dot{e}_{3}^{T}(t) \bar{P}_{03} e_{3}(t)+e_{3}^{T}(t) \bar{P}_{03} \dot{e}_{3}(t) \\
= & \sum_{i=1}^{k} \mu_{i}(\theta(t))\left\{e_{1}^{T}(t)\left(\left(A_{1, i}^{s}\right)^{T} P_{1}+P_{1} A_{1, i}^{s}\right) e_{1}(t)\right. \\
+ & e_{2}^{T}(t)\left(\left(A_{4, i}+L C_{4, i}\right)^{T} P_{01}+P_{01}\left(A_{4, i}+L C_{4, i}\right)\right) \\
\cdot & e_{2}(t)+e_{3}^{T}(t)\left(\bar{P}_{03} K_{i}+K_{i}^{T} \bar{P}_{03}\right) e_{3}(t)+2 e_{1}^{T}(t) \\
\cdot & P_{1} A_{2, i} e_{2}(t)+2 e_{3}^{T}(t) \bar{P}_{03} C_{4, i} e_{2}(t)+2 e_{1}^{T}(t) \\
& \left.\cdot P_{1} D_{1, i} \xi(t)+2 e_{2}^{T}(t) P_{01} D_{2, i} \xi(t)\right\} .
\end{aligned}
$$

In order to achieve the robustness of the proposed sliding mode observers to the disturbances $\xi(t)$ in the $L_{2}$ sense, we define

$$
J(t):=\dot{V}(t)+g^{T}(t) g(t)-\gamma \xi^{T}(t) \xi(t) .
$$

As a result, it yields that (41) is satisfied where, $\forall i \in$ $[1, \ldots, k]$,

$$
\begin{aligned}
Q_{1, i} & =\left(A_{1, i}^{s}\right)^{T} P_{1}+P_{1} A_{1, i}^{s}, \\
Q_{2, i} & =A_{4, i}^{T} P_{01}+P_{01} A_{4, i}+P_{01} L C_{4, i}+C_{4, i}^{T} L^{T} P_{01}, \\
Q_{3, i} & =\bar{P}_{03} K_{i}+K_{i}^{T} \bar{P}_{03} .
\end{aligned}
$$

If $\Delta_{i}<0, \forall i \in[1, \ldots, k]$, then $J(t) \leq 0$ along system trajectories with disturbances attenuation level $\gamma$ : 


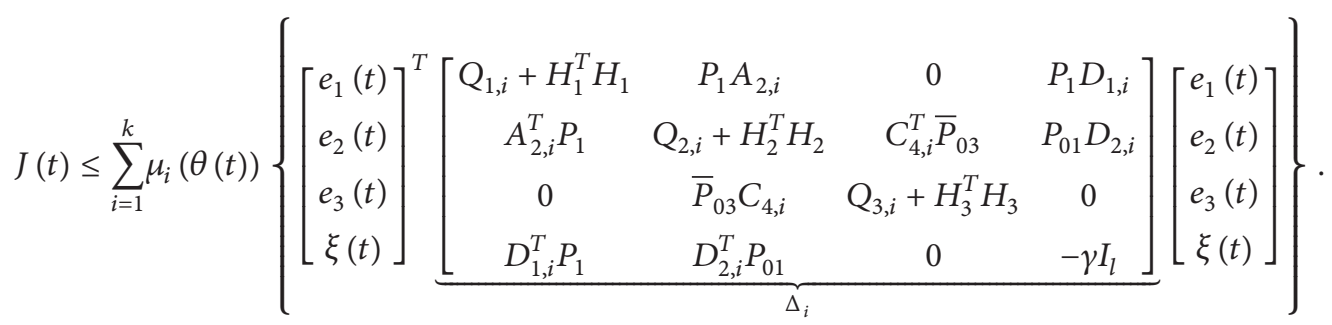

Integrating the expression in (39) from 0 to $\infty$, we can obtain with respect to $t$ over time period $\left[\begin{array}{ll}0 & \infty\end{array}\right]$ the following:

$$
\int_{0}^{\infty} \dot{V}(t)+\int_{0}^{\infty} g^{T}(t) g(t)-\int_{0}^{\infty} \gamma \xi^{T}(t) \xi(t)<0,
$$

together with

$$
\begin{aligned}
V(t=0)>0 & \\
V(t \longrightarrow \infty)= & e_{1}^{T}(\infty) P_{1} e_{1}(\infty)+e_{2}^{T}(\infty) P_{01} e_{2}(\infty) \\
& +e_{3}^{T}(\infty) \bar{P}_{03} e_{3}(\infty) \longrightarrow 0 .
\end{aligned}
$$

Therefore, we obtain

$$
\frac{\int_{0}^{\infty} g^{T}(t) g(t)}{\int_{0}^{\infty} \xi^{T}(t) \xi(t)} \leq \gamma,
$$

namely,

$$
\|g(t)\|_{L 2} \leq \sqrt{\gamma}\|\xi(t)\|_{L 2} .
$$

This completes the proof.

3.2. Sliding Motion Reachability. For systems (24)-(26), consider the sliding surface

$$
S_{g}=\left\{\left(e_{1}(t), e_{2}(t), e_{3}(t)\right) \mid e_{1}(t)=0, e_{3}(t)=0\right\} .
$$

In order to confine stable sliding motion for the polytopic LPV system on sliding surface, it is necessary to use the switching gains $v_{1, i}(t)$ and $v_{2, i}(t)$. The objective now is to determine the scalars gains $\eta_{1, i}$ in (22) and $\eta_{2, i}$ in (23) such that the errors system can be driven to the sliding surface $S_{g}$ in finite time.

The main challenge of this subsection will be proved.

Theorem 4. The error dynamics (24) and (26) can be driven to the sliding surface $S_{g}(46)$ in finite time and remain on it if the gains $\eta_{1, i}$ and $\eta_{2, i}$ are chosen to satisfy

$$
\begin{aligned}
& \eta_{1, i} \geq\left\|M_{1, i}^{-T}\right\|\left(\left\|A_{2, i}\right\|\left\|e_{2}(t)\right\|+\left\|D_{2, i}\right\| \xi_{0}\right)+\eta_{1, i}^{0}, \\
& \eta_{2, i} \geq\left\|N_{2, i}^{-T}\right\|\left\|C_{4, i}\right\|\left\|e_{2}(t)\right\|+\eta_{2, i}^{0},
\end{aligned}
$$

where $\eta_{1, i}^{0}$ and $\eta_{2, i}^{0}$ are positive scalars $\forall i \in[1, \ldots, k]$.
Proof. Let $V_{1}(t)=e_{1}^{T}(t) P_{1} e_{1}(t)$. From the expression of the estimation error in (24), it turns out that

$$
\begin{aligned}
& \dot{V}_{1}(t)=\sum_{i=1}^{k} \mu_{i}(\theta(t)) \\
& \cdot\left\{e_{1}^{T}(t)\left(\left(A_{1, i}^{s}\right)^{T} P_{1}+P_{1} A_{1, i}^{s}\right) e_{1}(t)\right. \\
& +2 e_{1}^{T}(t) P_{1} A_{2, i} e_{2}(t)+2 e_{1}^{T}(t) P_{1} D_{1, i} \xi(t) \\
& \left.\quad+2 e_{1}^{T}(t) P_{1} M_{1, i} f_{a}(t)-2 e_{1}^{T}(t) P_{1} M_{1, i} v_{1, i}(t)\right\} .
\end{aligned}
$$

Since by design $A_{1, i}^{s}$ is stable matrices, $\forall i \in[1, \ldots, k]$, it follows that $\left(A_{1, i}^{s}\right)^{T} P_{1}+P_{1} A_{1, i}^{s}<0$. Then, from the CauchySchwartz inequality and by applying (22), we can obtain that

$$
\begin{aligned}
& \dot{V}_{1}(t) \leq \sum_{i=1}^{k} \mu_{i}(\theta(t))\left\{2 e_{1}^{T}(t) P_{1}\left(A_{2, i} e_{2}(t)+D_{1, i} \xi(t)\right)\right. \\
& \left.+2 e_{1}^{T}(t) P_{1} M_{1, i} f_{a}(t)-2 e_{1}^{T}(t) P_{1} M_{1, i} \nu_{1, i}(t)\right\} \\
& \quad \leq \sum_{i=1}^{k} \mu_{i}(\theta(t))\left\{2\left\|P_{1} e_{1}(t)\right\|\left\|A_{2, i}\right\|\left\|e_{2}(t)\right\|\right. \\
& +2\left\|P_{1} e_{1}(t)\right\|\left\|D_{1, i}\right\|\|\xi(t)\|+2 e_{1}^{T}(t) P_{1} M_{1, i} f_{a}(t) \\
& \quad-2\left(\rho_{1}+\eta_{1, i}\right) e_{1}^{T}(t) P_{1} M_{1, i} \frac{M_{1, i}^{T} P_{1} e_{1}(t)}{\left.\left\|M_{1, i}^{T} P_{1} e_{1}(t)\right\|\right\}} \\
& \quad \leq \sum_{i=1}^{k} \mu_{i}(\theta(t))\left\{2\left\|P_{1} e_{1}(t)\right\|\left\|A_{2, i}\right\|\left\|e_{2}(t)\right\|\right. \\
& +2\left\|P_{1} e_{1}(t)\right\|\left\|D_{1, i}\right\|\|\xi(t)\|+2\left\|M_{1, i}^{T} P_{1} e_{1}(t)\right\| \rho_{1} \\
& \quad-2\left(\rho_{1}+\eta_{1, i}\right) \frac{\left\|M_{1, i}^{T} P_{1} e_{1}(t)\right\| \|^{2}}{\left.\left\|M_{1, i}^{T} P_{1} e_{1}(t)\right\|\right\} \leq \sum_{i=1}^{k} \mu_{i}(\theta(t))} \\
& \quad \cdot\left\{2\left\|P_{1} e_{1}(t)\right\|\left(\left\|A_{2, i}\right\|\left\|e_{2}(t)\right\|+\left\|D_{1, i}\right\|\|\xi(t)\|\right)\right. \\
& \left.\quad-2 \eta_{1, i}\left\|M_{1, i}^{T} P_{1} e_{1}(t)\right\|\right\} .
\end{aligned}
$$


It follows from (47) that

$$
\begin{aligned}
\dot{V}_{1}(t) & \leq \sum_{i=1}^{k} \mu_{i}(\theta(t))\left\{-2 \eta_{1, i}^{0}\left\|M_{1, i}^{T} P_{1} e_{1}(t)\right\|\right\} \\
& \leq \sum_{i=1}^{k} \mu_{i}(\theta(t))\left\{-2 \eta_{1, i}^{0}\left\|M_{1, i}^{T}\right\| \sqrt{\lambda_{\min }\left(P_{1}\right)}\right\} V_{1}^{(1 / 2)} .
\end{aligned}
$$

Similarly, it can be verified from (23) that if (48) is satisfied, then

$$
\begin{aligned}
\dot{V}_{3}(t) & \leq \sum_{i=1}^{k} \mu_{i}(\theta(t))\left\{-2 \eta_{2, i}^{0}\left\|N_{2, i}^{T} \bar{P}_{03} e_{3}(t)\right\|\right\} \\
& \leq \sum_{i=1}^{k} \mu_{i}(\theta(t))\left\{-2 \eta_{2, i}^{0}\left\|N_{2, i}^{T}\right\| \sqrt{\lambda_{\min }\left(\bar{P}_{03}\right)}\right\} V_{3}^{(1 / 2)} .
\end{aligned}
$$

Thus, it shows that the reachability condition is satisfied and an ideal sliding motion is achieved and maintained after some finite time $t_{f} \geq 0$.

This completes the proof.

\section{Simultaneous Actuator and Sensor Faults Estimation}

It is assumed in this section that the sliding mode observers (20)-(21) have been designed. The objective is to simultaneously estimate actuator and sensor faults. From Theorems 3 and 4, it follows that, in finite time, the error dynamics (24) and (26) will be driven to the sliding surface $S_{g}$ and an ideal sliding motion is maintained therefore. During the sliding motion,

$$
\begin{aligned}
& e_{1}(t)=\dot{e}_{1}(t)=0, \\
& e_{3}(t)=\dot{e}_{3}(t)=0 .
\end{aligned}
$$

One can prove that (24) becomes

$$
\begin{aligned}
0= & \sum_{i=1}^{k} \mu_{i}(\theta(t))\left\{A_{2, i} e_{2}(t)+D_{1, i} \xi(t)+M_{1, i} f_{a}(t)\right. \\
& \left.-M_{1, i} v_{1_{\text {eq }}, i}(t)\right\}
\end{aligned}
$$

where $v_{1_{\mathrm{eq}}, i}(t)$ denotes the equivalent output error injection signal required to maintain the sliding motion [2]. The signal $v_{1_{\mathrm{eq}}, i}(t)$ can be approximated by

$$
\begin{aligned}
v_{1_{\mathrm{eq}}, i}(t)= & \left(\rho_{1}+\eta_{1, i}\right) \\
& \times \frac{M_{1, i}^{T} P_{1}\left(C_{1, i}^{-1} S_{1, i} y(t)-\widehat{x}_{1}(t)\right)}{\left\|M_{1, i}^{T} P_{1}\left(C_{1, i}^{-1} S_{1, i} y(t)-\widehat{x}_{1}(t)\right)\right\|+\delta_{1}},
\end{aligned}
$$

where $\delta_{1}$ is a small positive scalar designed to reduce the chattering effect.
It remains to prove, from (54), that $\forall i \in[1, \ldots, k]$,

$$
\begin{gathered}
\left\|f_{a}(t)-v_{1_{\text {eq }}, i}(t)\right\|_{L_{2}} \\
=\left\|M_{1, i}^{-1} A_{2, i} e_{2}(t)+M_{1, i}^{-1} D_{1, i} \xi(t)\right\|_{L_{2}} \\
\leq \sigma_{\max }\left(M_{1, i}^{-1} A_{2, i}\right)\left\|e_{2}(t)\right\|_{L_{2}} \\
\quad+\sigma_{\max }\left(M_{1, i}^{-1} D_{1, i}\right)\|\xi(t)\|_{L_{2}} \\
\leq \sigma_{\max }\left(M_{1, i}^{-1} A_{2, i}\right)\|e(t)\|_{L_{2}} \\
\quad+\sigma_{\max }\left(M_{1, i}^{-1} D_{1, i}\right)\|\xi(t)\|_{L_{2}}
\end{gathered}
$$

So, since $\|e(t)\|_{L 2} \leq \sigma_{\max }\left(H^{-1}\right) \sqrt{\gamma}\|\xi(t)\|_{L 2}$,

$$
\sup _{\|\xi(t)\|_{L_{2}} \neq 0} \frac{\left\|f_{a}(t)-v_{1_{\text {eq }}, i}(t)\right\|_{L_{2}}}{\|\xi(t)\|_{L_{2}}}=\sqrt{\gamma} \beta_{11, i}+\beta_{12, i}
$$

where $\beta_{11, i}=\sigma_{\max }\left(M_{1, i}^{-1} A_{2, i}\right) \sigma_{\max }\left(H^{-1}\right)$ and $\beta_{12, i}=$ $\sigma_{\max }\left(M_{1, i}^{-1} D_{1, i}\right), \forall i \in[1, \ldots, k]$.

Thus, for small $\beta_{11, i}$ and $\beta_{12, i}, \forall i \in[1, \ldots, k]$, the actuator fault signal can be obtained as

$$
\begin{aligned}
\widehat{f}_{a}(t) & \cong \sum_{i=1}^{k} \mu_{i}(\theta(t))\left\{\left(\rho_{1}+\eta_{1, i}\right)\right. \\
& \left.\times \frac{M_{1, i}^{T} P_{1}\left(C_{1, i}^{-1} S_{1, i} y(t)-\widehat{x}_{1}(t)\right)}{\left\|M_{1, i}^{T} P_{1}\left(C_{1, i}^{-1} S_{1, i} y(t)-\widehat{x}_{1}(t)\right)\right\|+\delta_{1}}\right\} .
\end{aligned}
$$

Similarly, we can deduce that

$$
\begin{aligned}
\left\|f_{s}(t)-v_{2_{\mathrm{eq}}, i}(t)\right\|_{L_{2}} & =\left\|N_{2, i}^{-1} C_{4, i} e_{2}(t)\right\|_{L_{2}} \\
& \leq \sigma_{\max }\left(N_{2, i}^{-1} C_{4, i}\right)\left\|e_{2}(t)\right\|_{L_{2}} \\
& \leq \sigma_{\max }\left(N_{2, i}^{-1} C_{4, i}\right)\|e(t)\|_{L_{2}},
\end{aligned}
$$

namely,

$$
\sup _{\|\xi(t)\|_{L_{2}} \neq 0} \frac{\left\|f_{s}(t)-v_{2_{\mathrm{eq}}, i}(t)\right\|_{L_{2}}}{\|\xi(t)\|_{L_{2}}}=\sqrt{\gamma} \beta_{2, i}
$$

where $\beta_{2, i}=\sigma_{\max }\left(N_{2, i}^{-1} C_{4, i}\right) \sigma_{\max }\left(H^{-1}\right)$ will be small scalars, $\forall i \in[1, \ldots, k]$.

Therefore, the sensor fault estimation is handled as follows:

$$
\begin{aligned}
& \widehat{f}_{s}(t) \cong \sum_{i=1}^{k} \mu_{i}(\theta(t)) \\
& \cdot\left\{\left(\rho_{2}+\eta_{2, i}\right) \times \frac{N_{2, i}^{T} \bar{P}_{03}\left(y_{0}(t)-\widehat{y}_{0}(t)\right)}{\left\|N_{2, i}^{T} \bar{P}_{03}\left(y_{0}(t)-\widehat{y}_{0}(t)\right)\right\|+\delta_{2}}\right\},
\end{aligned}
$$

where $\delta_{2}$ is a small positive scalar. 


\section{Illustrative Example}

5.1. Model Expression. The performances of the proposed sliding mode observer-based simultaneous actuator and sensor faults estimation is performed by considering a polytopic LPV representation of the Vertical Take-Off and Landing (VTOL) Aircraft defense system taken from [31]. The LPV state-space model is given by

$$
\begin{aligned}
\dot{x}(t)= & A(\theta(t)) x(t)+B(\theta(t)) u(t)+D(\theta(t)) \xi(t) \\
& +M(\theta(t)) f_{a}(t), \\
y(t)= & C(\theta(t)) x(t)+N(\theta(t)) f_{s}(t),
\end{aligned}
$$

where the state vector $x(t)=\left[\begin{array}{llll}V_{h} & V_{f} & q & \varphi\end{array}\right]^{T}$ represents, respectively, horizontal velocity, vertical velocity, pitch rate, and pitch angle. $u(t)=\left[\begin{array}{ll}u_{c} & u_{l}\end{array}\right]^{T}$ are, respectively, pitch angle, collective pitch control, and longitudinal cyclic pitch control. $\xi(t)$ denotes unknown external disturbances vector. It is worth pointing that simulation results are illustrated with simultaneous additive faults $f_{s}(t)$ affecting measurement outputs and actuator faults $f_{a}(t)$. The LPV system matrices are expressed as

$$
\begin{aligned}
& A(\theta(t)) \\
& =\left[\begin{array}{cccc}
-9.9477 & -0.7476 & 0.2632 & 5.0337 \\
52.1659 & 2.7452 & 5.5532 & -24.4221 \\
26.0922 & 2.6361+\theta_{1}(t) & -4.1975 & -19.2774+\theta_{2}(t) \\
0 & 0 & 1 & 0
\end{array}\right], \\
& B(\theta(t))=\left[\begin{array}{cc}
0.4422 & 0.1761 \\
3.5446+\theta_{2}(t) & -7.5922 \\
-5.5200 & 4.4900 \\
0 & 0
\end{array}\right], \\
& D(\theta(t))=D=\left[\begin{array}{c}
0.1 \\
0 \\
0.1 \\
0.1
\end{array}\right], \\
& N(\theta(t))=N=\left[\begin{array}{ll}
0.1761 \\
-7.5922 \\
4.4900 \\
0 \\
1
\end{array}\right], \\
& M(\theta(t))=M=C=\left[\begin{array}{ccc}
1 & 0 \\
0 & 1 & 0 \\
0 & 0 & 1
\end{array}\right],
\end{aligned}
$$

The varying parameter functions $\theta_{1}(t)$ and $\theta_{2}(t)$ are bounded as $\theta_{1}(t) \in[-0.5,0.5]$ and $\theta_{2}(t) \in[-2,2]$. Therefore, corresponding to the extreme values of the parameters $\theta_{1}(t)$ and $\theta_{2}(t)$, an obtained system evolves in a polytope of 4 vertices described as (5). The polytopic LPV system matrices are given as

$$
A_{1}=\left[\begin{array}{cccc}
-9.9477 & -0.7476 & 0.2632 & 5.0337 \\
52.1659 & 2.7452 & 5.5532 & -24.4221 \\
26.0922 & 2.1361 & -4.1975 & -21.2774 \\
0 & 0 & 1 & 0
\end{array}\right],
$$$$
A_{2}=\left[\begin{array}{cccc}
-9.9477 & -0.7476 & 0.2632 & 5.0337 \\
52.1659 & 2.7452 & 5.5532 & -24.4221 \\
26.0922 & 2.1361 & -4.1975 & -17.2774 \\
0 & 0 & 1 & 0
\end{array}\right] \text {, }
$$$$
A_{3}=\left[\begin{array}{cccc}
-9.9477 & -0.7476 & 0.2632 & 5.0337 \\
52.1659 & 2.7452 & 5.5532 & -24.4221 \\
26.0922 & 3.1361 & -4.1975 & -21.2774 \\
0 & 0 & 1 & 0
\end{array}\right] \text {, }
$$$$
A_{4}=\left[\begin{array}{cccc}
-9.9477 & -0.7476 & 0.2632 & 5.0337 \\
52.1659 & 2.7452 & 5.5532 & -24.4221 \\
26.0922 & 3.1361 & -4.1975 & -17.2774 \\
0 & 0 & 1 & 0
\end{array}\right] \text {, }
$$$$
B_{1}=\left[\begin{array}{cc}
0.4422 & 0.1761 \\
1.5446 & -7.5922 \\
-5.5200 & 4.4900 \\
0 & 0
\end{array}\right]
$$

$$
B_{2}=\left[\begin{array}{cc}
0.4422 & 0.1761 \\
5.5446 & -7.5922 \\
-5.5200 & 4.4900 \\
0 & 0
\end{array}\right] \text {, }
$$$$
B_{3}=\left[\begin{array}{cc}
0.4422 & 0.1761 \\
1.5446 & -7.5922 \\
-5.5200 & 4.4900 \\
0 & 0
\end{array}\right] \text {, }
$$$$
B_{4}=\left[\begin{array}{cc}
0.4422 & 0.1761 \\
5.5446 & -7.5922 \\
-5.5200 & 4.4900 \\
0 & 0
\end{array}\right] \text {. }
$$

The convex weighing functions $\mu_{i}(\theta(t))$ are

$$
\begin{aligned}
\mu_{1}(\theta(t)) & =\frac{\theta_{1}(t)-\underline{\theta}_{1}}{\bar{\theta}_{1}-\underline{\theta}_{1}} \frac{\underline{\theta}_{2}(t)-\underline{\theta}_{2}}{\bar{\theta}_{2}-\underline{\theta}_{2}} \\
& =\frac{\left(\theta_{1}(t)+0.5\right)\left(\theta_{2}(t)+2\right)}{4},
\end{aligned}
$$




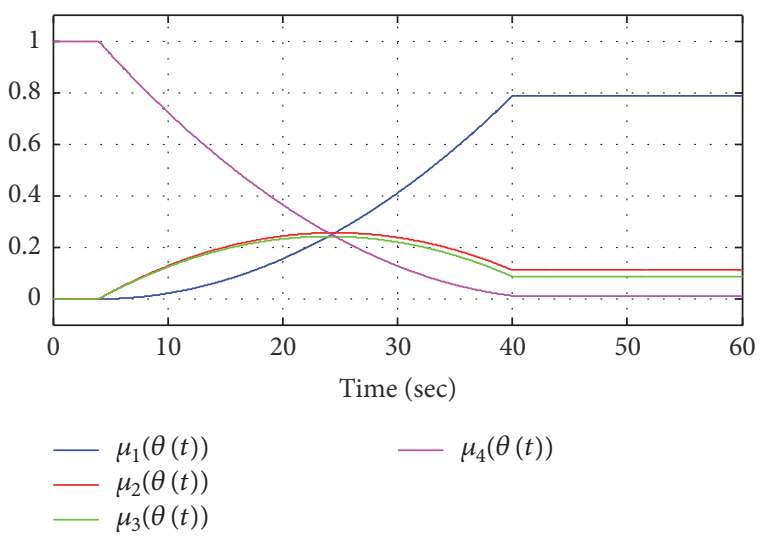

FIGURE 1: Weighing functions.

$$
\begin{aligned}
\mu_{2}(\theta(t)) & =\frac{\theta_{1}(t)-\underline{\theta}_{1}}{\bar{\theta}_{1}-\underline{\theta}_{1}} \frac{\bar{\theta}_{2}-\theta_{2}(t)}{\bar{\theta}_{2}-\underline{\theta}_{2}} \\
& =\frac{\left(\theta_{1}(t)+0.5\right)\left(-\theta_{2}(t)+2\right)}{4}, \\
\mu_{3}(\theta(t)) & =\frac{\bar{\theta}_{1}-\theta_{1}(t)}{\bar{\theta}_{1}-\underline{\theta}_{1}} \frac{\theta_{2}(t)-\underline{\theta}_{2}}{\bar{\theta}_{2}-\underline{\theta}_{2}} \\
& =\frac{\left(0.5-\theta_{1}(t)\right)\left(\theta_{2}(t)+2\right)}{4}, \\
\mu_{4}(\theta(t)) & =\frac{\bar{\theta}_{1}-\theta_{1}(t)}{\bar{\theta}_{1}-\underline{\theta}_{1}} \frac{\bar{\theta}_{2}-\theta_{2}(t)}{\bar{\theta}_{2}-\underline{\theta}_{2}} \\
& =\frac{\left(0.5-\theta_{1}(t)\right)\left(2-\theta_{2}(t)\right)}{4} .
\end{aligned}
$$

These functions of each local model are depicted in Figure 1. that

Based on the polytopic LPV system matrices, it follows

(i) $\operatorname{Rank}(C M)=\operatorname{Rank}(M)=1$;

(ii) the invariant zeros of the triplet system $\left(A_{i}, M, C\right)$ are in the open left-hand complex plane.

Therefore, Assumptions A1 and A2 are satisfied.

5.2. Simulation Results. The aim is to design the proposed $H_{\infty}$ sliding mode observers-based actuator and sensor faults estimation in the simultaneous scenario assuming that the matrix $H$ is chosen as $H_{1}=1, H_{2}=I_{3}$, and $H_{3}=I_{2}$.

The disturbances attenuation level is determined as $\gamma=$ 0.3720 . By solving the LMI conditions given in Theorem 3, the sliding mode observers (20)-(21) design is achieved as follows:

$$
\begin{aligned}
& A_{1,1}^{s}=-12.547, \\
& A_{1,2}^{s}=-12.145,
\end{aligned}
$$

$$
\begin{aligned}
& A_{1,3}^{s}=-12.557, \\
& A_{1,4}^{s}=-12.169 \text {, } \\
& P_{1}=0.184 \text {, } \\
& P_{01}=\left[\begin{array}{ccc}
0.153 & 0.033 & -0.4 \\
0.033 & 0.107 & -0.505 \\
-0.4 & -0.505 & 10.748
\end{array}\right] \text {, } \\
& \bar{P}_{03}=\left[\begin{array}{cc}
0.863 & 0 \\
0 & 0.604
\end{array}\right] \text {, } \\
& L=\left[\begin{array}{cc}
10.29 & 0 \\
-53.064 & 0 \\
-4.5 & 0
\end{array}\right] \text {, } \\
& K_{1}=\left[\begin{array}{cc}
-2.35 & 0.057 \\
0.077 & -3.481
\end{array}\right] \text {, } \\
& K_{2}=\left[\begin{array}{cc}
-2.341 & 0.084 \\
0.115 & -3.4
\end{array}\right] \text {, } \\
& K_{3}=\left[\begin{array}{cc}
-2.413 & 0.014 \\
0.015 & -3.487
\end{array}\right] \text {, } \\
& K_{4}=\left[\begin{array}{cc}
-2.391 & 0.044 \\
0.058 & -3.422
\end{array}\right] \text {. }
\end{aligned}
$$

The corresponding simulations are done by considering initial conditions $x(t=0)=[0 ; 0 ; 0 ; 0]$ and $\widehat{x}(t=0)=$ $[-0.2 ; 1 ; 1 ;-0.25]$, respectively, for the LPV system and observers. We assume also $\rho_{1}=10, \rho_{2}=20, \delta_{1}=0.05, \delta_{2}=$ 0.02 and simultaneous actuator and sensor faults scenario to leave $t=5 \mathrm{~s}$. The simulation was carried out with the input signal $u(t)=2 \sin (t)$ and uncertainty $\xi(t)=0.1 \sin (0.2 t)$.

Figure 2 displays the states estimation errors to prove a comparison between $x_{1}(t), x_{2}(t), x_{3}(t)$, and $x_{4}(t)$ against the estimated values $\widehat{x}_{1}(t), \widehat{x}_{2}(t), \widehat{x}_{3}(t)$, and $\widehat{x}_{4}(t)$ respectively. It can be seen that the estimated states can converge towards the original states.

In order to highlight the effectiveness of the proposed robust and simultaneous actuator and sensor faults estimation for LPV systems compared to descriptor approach [29], we consider different scenarios of faults occurrence.

5.2.1. Simulation Results under Incipient Actuator Faults. The objective of the first scenario is to illustrate the sliding mode observer performances under an incipient actuator fault. Incipient faults are important in nonlinear complex system, and some common manifestations include zero drift, reduced precision, and delayed response. Consequently, the study of incipient faults in nonlinear systems has practical significance.

As can be observed, the proposed sliding mode observers are capable of estimating incipient actuator fault after a short 


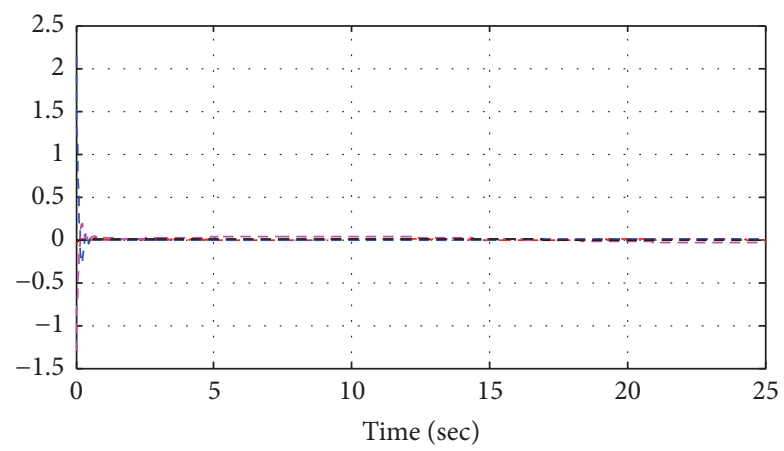

- - - Horizontal velocity estimation error

- - - Vertical velocity estimation error

- - - Pitch rate estimation error

- - - Pitch angle estimation error

FIGURE 2: States estimation errors.

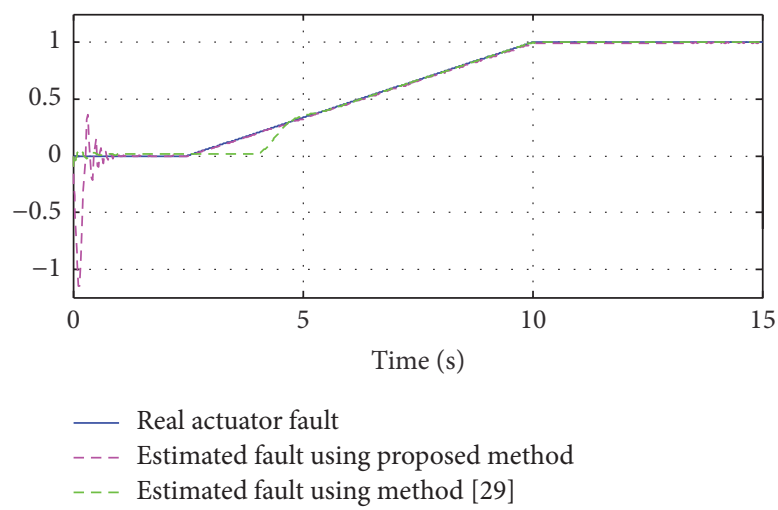

FIgURE 3: Actuator fault estimation under incipient actuator fault.

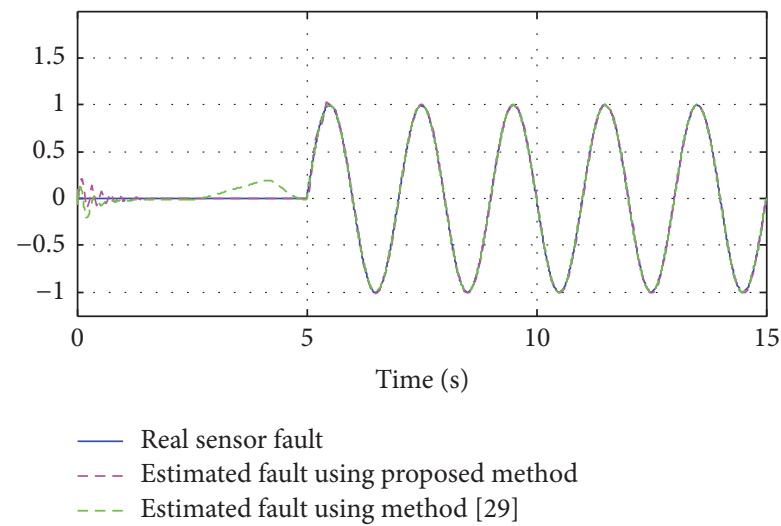

FIGURE 4: Sensor fault estimation under incipient actuator fault.

time of fault generation, as well as sensor faults (sinusoidal signal) with good performances and small error compared to the estimation based on descriptor approach [29]. So, one can see, from Figures 3 and 4, that the external disturbances are faithfully reduced by integrating the $H_{\infty}$ attenuation levels into the sliding mode observers design.

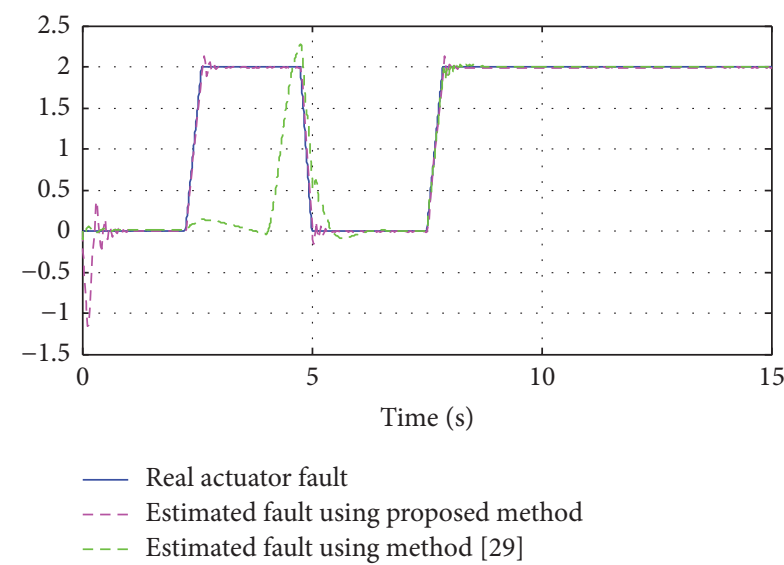

FIGURE 5: Actuator fault estimation under intermittent actuator fault.

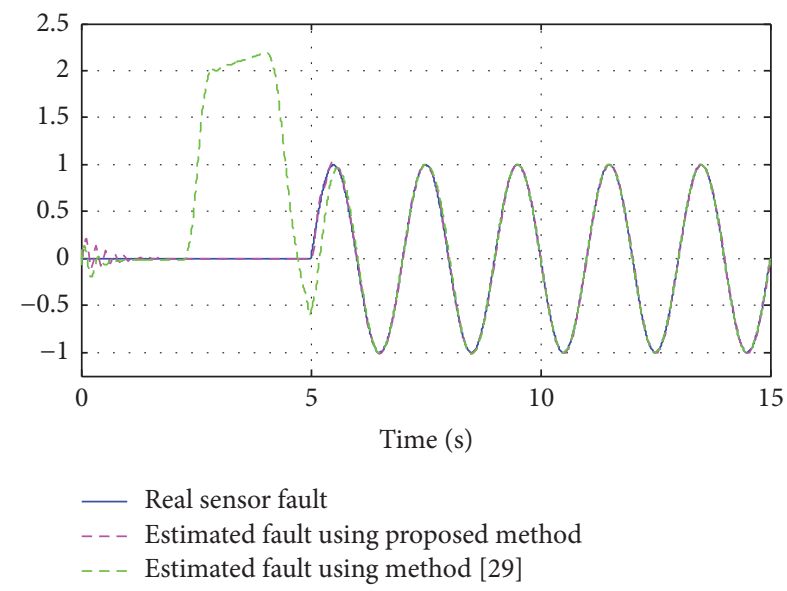

FIGURE 6: Sensor fault estimation under intermittent actuator fault.

5.2.2. Simulation Results under Intermittent Actuator Faults. The second scenario has the objective of illustrating the proposed sliding mode observers performances under intermittent actuator faults. Figures 5 and 6 show simultaneous actuator and sensor faults estimation under intermittent actuator faults. The results display that the proposed sliding mode observers present better performances under intermittent actuator fault compared to descriptor approach [29]. The proposed method is capable of decoupling the effects of simultaneous actuator and sensor faults and managing to accomplish the main objective of this paper (both actuator and sensor fault estimation for the polytopic LPV system) with good performances in terms of precision and robustness against the external disturbances.

\section{Conclusion}

This paper presents a robust simultaneous actuator and sensor faults estimation scheme for LPV systems with disturbances. The developed strategy is based on polytopic representation where the parameters evolve in the hypercube domain. State and output transformations are introduced in 
order to formulate two polytopic LPV subsystems where each one includes a particular class of faults (actuator or sensor). To this end, two sliding mode observers are conceived to exactly estimate simultaneously: incipient and intermittent actuator faults, sensor faults, and polytopic LPV system states. Sufficient conditions to guarantee the stability of the two observers has been derived with $H_{\infty}$ performances for minimizing the effect of disturbances on estimation error dynamics and solved within LMIs optimization design. The simulation results display that the proposed scheme can successfully estimate actuator and sensor faults simultaneously despite the existence of disturbances.

\section{Conflicts of Interest}

The authors declare that they have no conflicts of interest.

\section{References}

[1] K. A. Hesam and Y. Alireza, "A new robust $H_{\infty}$ sliding mode observer-based state estimation and fault reconstruction for nonlinear uncertain boiler system," Soft Computing, pp. 1-12, 2016.

[2] C. Edwards, S. K. Spurgeon, and R. J. Patton, "Sliding mode observers for fault detection and isolation," Automatica, vol. 36, no. 4, pp. 541-553, 2000.

[3] K. Y. Ng, C. P. Tan, C. Edwards, and Y. C. Kuang, "New results in robust actuator fault reconstruction for linear uncertain systems using sliding mode observers," International Journal of Robust and Nonlinear Control, vol. 17, no. 14, pp. 1294-1319, 2007.

[4] S. Dhahri, A. Sellami, and F. B. Hmida, "Robust $H_{\infty}$ sliding mode observer design for fault estimation in a class of uncertain nonlinear systems with LMI optimization approach," International Journal of Control, Automation, and Systems, vol. 10, no. 5, pp. 1032-1041, 2012.

[5] R. Raoufi, H. J. Marquez, and A. S. Zinober, " $H_{\infty}$ sliding mode observers for uncertain nonlinear Lipschitz systems with fault estimation synthesis," International Journal of Robust and Nonlinear Control, vol. 20, no. 16, pp. 1785-1801, 2010.

[6] X.-G. Yan and C. Edwards, "Nonlinear robust fault reconstruction and estimation using a sliding mode observer," Automatica, vol. 43, no. 9, pp. 1605-1614, 2007.

[7] J. Yang, Y. Chen, and K. Yu, "State and unknown information estimation for non-linear systems with both input uncertainty and output disturbance," IMA Journal of Mathematical Control and Information, vol. 33, no. 2, pp. 427-439, 2014.

[8] J. Zhang, M. Lyu, H. R. Karimi, and Y. Bo, "Fault detection of networked control systems based on sliding mode observer," Mathematical Problems in Engineering, vol. 2013, Article ID 506217, 9 pages, 2013.

[9] C.-F. Zhang, M. Yan, J. He, and C. Luo, "LMI-based sliding mode observers for incipient faults detection in nonlinear system," Journal of Applied Mathematics, vol. 2012, Article ID 528932, 13 pages, 2012.

[10] J. He, L. Mi, S. Mao, C. Zhang, and H. Chu, "Fault-tolerant control of a nonlinear system actuator fault based on sliding mode control," Journal of Control Science and Engineering, vol. 2017, Article ID 8595960, 13 pages, 2017.

[11] M. Corless and J. Tu, "State and input estimation for a class of uncertain systems," Automatica, vol. 34, no. 6, pp. 757-764, 1998.
[12] C. P. Tan and C. Edwards, "Sliding mode observers for detection and reconstruction of sensor faults," Automatica, vol. 38, no. 10, pp. 1815-1821, 2002.

[13] H. Alwi, C. Edwards, and C. P. Tan, "Sliding mode estimation schemes for incipient sensor faults," Automatica, vol. 45, no. 7, pp. 1679-1685, 2009.

[14] X.-G. Yan and C. Edwards, "Sensor fault detection and isolation for nonlinear systems based on a sliding mode observer," International Journal of Adaptive Control and Signal Processing, vol. 21, no. 8-9, pp. 657-673, 2007.

[15] J. Zhang, A. K. Swain, and S. K. Nguang, "Detection and isolation of incipient sensor faults for a class of uncertain nonlinear systems," IET Control Theory \& Applications, vol. 6, no. 12, pp. 1870-1880, 2012.

[16] J. Zhang, A. K. Swain, and S. K. Nguang, "Robust sensor fault estimation scheme for satellite attitude control systems," Journal of The Franklin Institute, vol. 350, no. 9, pp. 2581-2604, 2013.

[17] M. J. Khosrowjerdi, "Robust sensor fault reconstruction for Lipschitz nonlinear systems," Mathematical Problems in Engineering, vol. 2011, Article ID 146038, 17 pages, 2011.

[18] M. Defoort, K. C. Veluvolu, J. J. Rath, and M. Djemai, "Adaptive sensor and actuator fault estimation for a class of uncertain Lipschitz nonlinear systems," International Journal of Adaptive Control and Signal Processing, vol. 30, no. 2, pp. 271-283, 2016.

[19] B. Zhao and Y. Li, "Multisensor fault identification scheme based on decentralized sliding mode observers applied to reconfigurable manipulators," Mathematical Problems in Engineering, vol. 2013, Article ID 327916, 9 pages, 2013.

[20] M. Sami and R. J. Patton, "Active fault tolerant control for nonlinear systems with simultaneous actuator and sensor faults," International Journal of Control, Automation, and Systems, vol. 11, no. 6, pp. 1149-1161, 2013.

[21] J. Zhang, A. K. Swain, and S. K. Nguang, "Simultaneous robust actuator and sensor fault estimation for uncertain non-linear Lipschitz systems," IET Control Theory \& Applications, vol. 8, no. 14, pp. 1364-1374, 2014.

[22] A. B. Brahim, S. Dhahri, F. B. Hmida, and S. Sellami, " $H_{\infty}$ Sliding Mode Observer for Takagi-Sugeno Nonlinear Systems with Simultaneous actuator and sensor faults," International Journal of Applied Mathematics and Computer Science, vol. 25, no. 3, pp. 547-559, 2015.

[23] A. B. Brahim, S. Dhahri, F. B. Hmida, and A. Sellami, "Simultaneous actuator and sensor faults reconstruction based on robust sliding mode observer for a class of nonlinear systems," Asian Journal of Control, vol. 19, no. 1, pp. 362-371, 2017.

[24] H. Hamdi, M. Rodrigues, C. Mechmeche, D. Theilliol, and N. B. Braiek, "State estimation for polytopic LPV descriptor systems: application to fault diagnosis," in Proceedings of the 7th IFAC Symposium on Fault Detection, Supervision and Safety of Technical Processes, Safeprocess '09, 2009.

[25] H. Hamdi, M. Rodrigues, C. Mechmeche, D. Theilliol, and N. B. Braiek, "Fault detection and isolation in linear parametervarying descriptor systems via proportional integral observer," International Journal of Adaptive Control and Signal Processing, vol. 26, no. 3, pp. 224-240, 2012.

[26] C. M. Astorga-Zaragoza, D. Theilliol, J. C. Ponsart, and M. Rodrigues, "Fault diagnosis for a class of descriptor linear parameter-varying systems," International Journal of Adaptive Control and Signal Processing, vol. 26, no. 3, pp. 208-223, 2012. 
[27] Z. Wang, M. Rodrigues, D. Theilliol, and Y. Shen, "Actuator fault estimation observer design for discrete-time linear parametervarying descriptor systems," International Journal of Adaptive Control and Signal Processing, vol. 29, no. 2, pp. 242-258, 2015.

[28] M. Rodrigues, H. Hamdi, D. Theilliol, C. Mechmeche, and N. B. Braiek, "Actuator fault estimation based adaptive polytopic observer for a class of LPV descriptor systems," International Journal of Robust and Nonlinear Control, vol. 25, no. 5, pp. 673688, 2014.

[29] X. Li and F. Zhu, "Simultaneous time-varying actuator and sensor fault reconstruction based on PI observer for LPV systems," International Journal of Adaptive Control and Signal Processing, vol. 29, no. 9, pp. 1086-1098, 2014.

[30] A. B. Brahim, S. Dhahri, F. B. Hmida, and A. Sellami, "Simultaneous actuator and sensor faults reconstruction using polytopic sliding mode observer for linear parameter varying systems," in Proceedings of the 14th International Conference on Sciences and Techniques of Automatic Control and Computer Engineering, STA '13, pp. 480-486, December 2013.

[31] Q. Jia, W. Chen, Y. Zhang, and X. Chen, "Fault reconstruction and accommodation in linear parameter-varying systems via learning unknown-input observers," Journal of Dynamic Systems, Measurement, and Control, vol. 137, no. 6, Article ID 061008, 2015. 


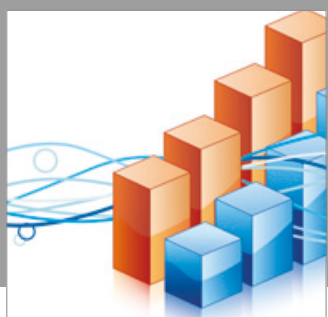

Advances in

Operations Research

vatersals

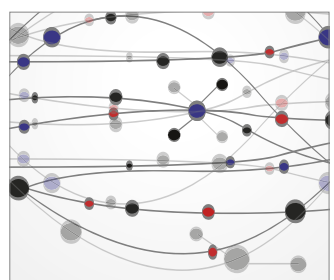

\section{The Scientific} World Journal
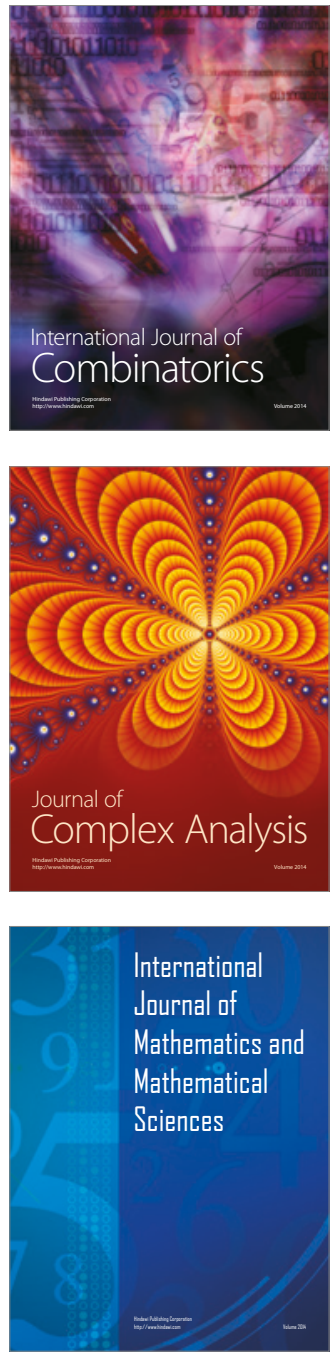
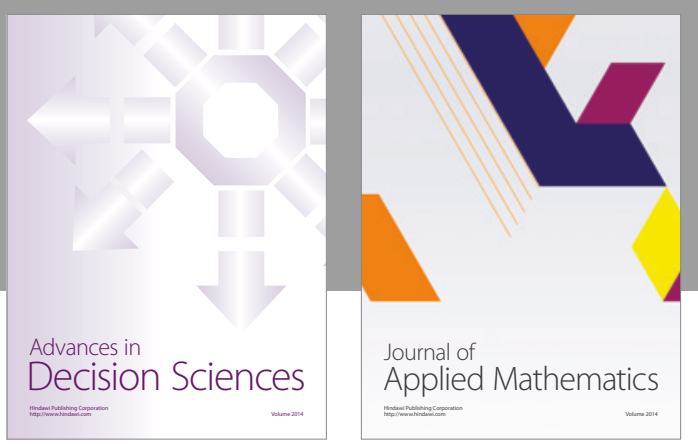

Algebra

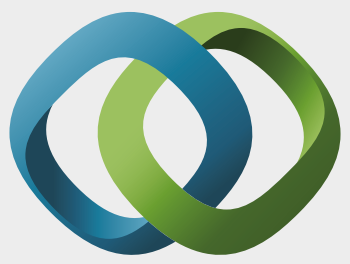

\section{Hindawi}

Submit your manuscripts at

https://www.hindawi.com
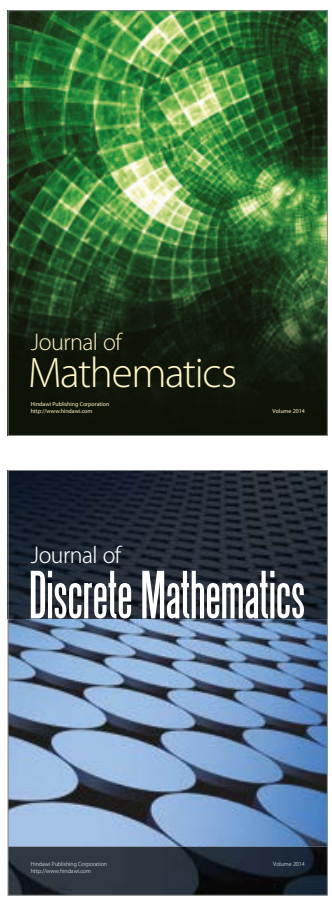

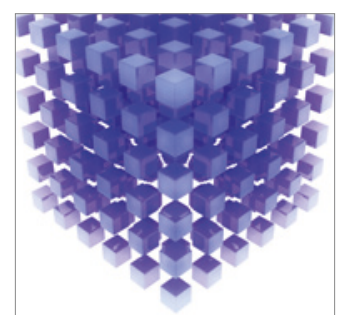

Mathematical Problems in Engineering
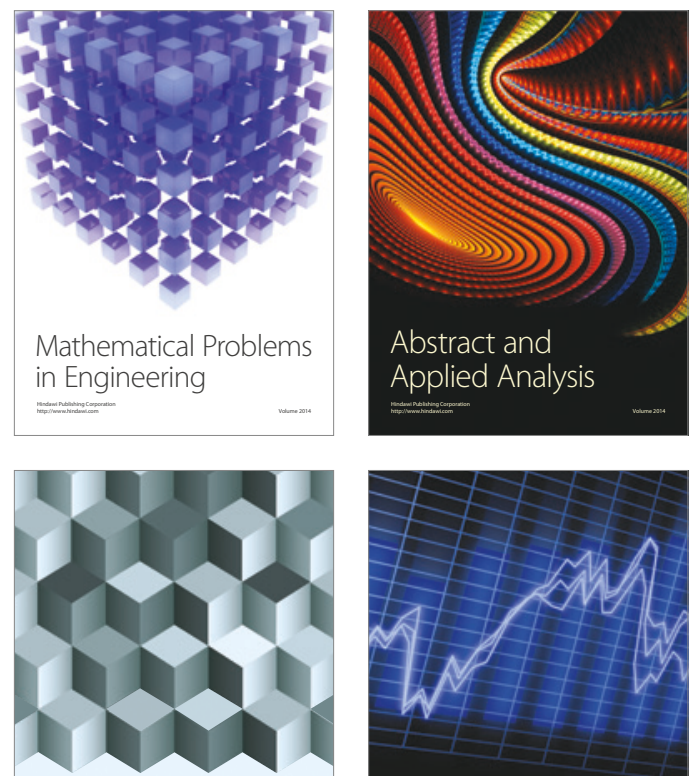

Journal of

Function Spaces

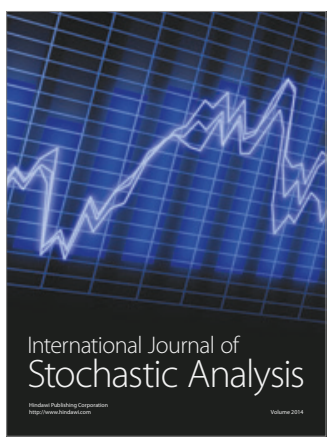

Probability and Statistics
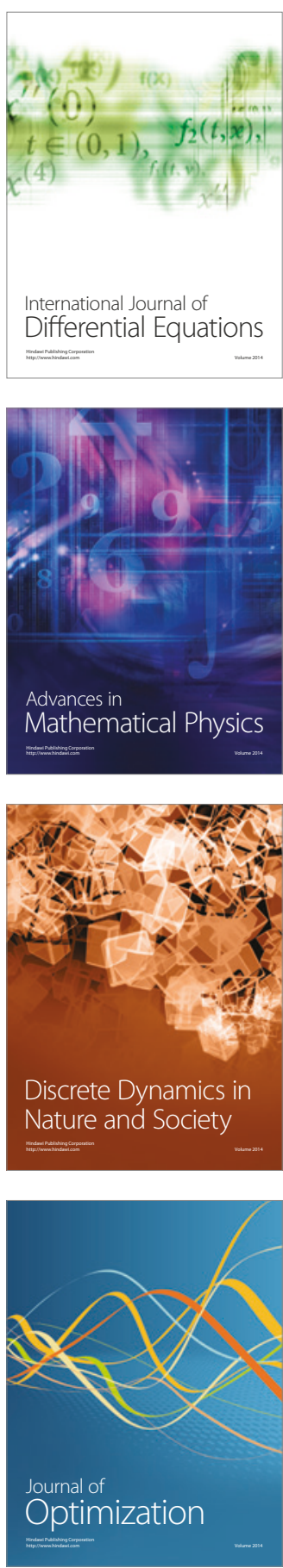